\title{
Perfil da demanda e morbidade dos pacientes atendidos em centro de urgências oftalmológicas de um hospital universitário
}

\author{
Demand profile and morbidity among patients treated \\ at service of ophthalmic urgencies in a university hospital
}

Frederico Braga Pereira', Maria Frasson², Ana Gabriela Zum Bach D'Almeida', Aline de Almeida ${ }^{3}$, Daniela Faria ${ }^{1}$, Júlia Francis ${ }^{4}$, João Neves de Medeiros ${ }^{5}$

\section{ResUmo}

Objetivo: Proporcionar análise epidemiológica dos pacientes atendidos no Serviço de Urgência Oftalmológica do Hospital São Geraldo. Métodos: Foi realizado estudo descritivo prospectivo no período de setembro de 2005 a janeiro de 2006 através de questionário de atendimento diário contendo sexo, idade, raça, ocupação e procedência dos pacientes, diagnóstico principal (de acordo com a Classificação Internacional de Doenças - 10 a revisão) e as condutas adotadas. Resultados: Foram atendidos 8.346 pacientes, 3.819 mulheres (45,79\%) e 4.521 homens (54,20\%). A faixa etária variou de 0 a 100 anos e o grupo de ocupações mais frequente foi o de serviços gerais $(24,20 \%)$. O diagnóstico mais frequente foi conjuntivite $(27,91 \%)$. A conduta mais adotada foi o tratamento medicamentoso $(65,5 \%)$. Conclusão: A maioria dos atendimentos foi classificada como urgência oftalmológica. As causas não traumáticas foram as mais incidentes. O Hospital São Geraldo desempenha um papel importante no atendimento à urgência oftalmológica da rede pública, na região de Belo Horizonte e cidades vizinhas.

Descritores: Oftalmopatias/epidemiologia; Emergências; Conjuntivite; Corpos estranhos no olho; Traumatismos oculares

\section{Abstract}

Objective: Establish an epidemiological analysis of patients assisted in an urgency service of a university eye hospital (Hospital São Geraldo). Methods: This is a prospective and descriptive study, conducted from september 2005 to january 2006. A questionnaire was used for the purpose of gathering daily information about all the patients assisted in the service. Information was collected on age, sex, occupation, ethnicity, origin of the patient, primary diagnosis (according to International Classification of Disease - ICD 10$)$ and the approaches adopted. Results: 8.346 patients were assisted, 3.819 female (45,79\%) and 4.521 male $(54,20 \%)$. The ages ranged from 0 to 100 years and the most common group of occupations was general services $(24,20 \%)$. The most frequent diagnosis was conjunctivitis $(27,91 \%)$. The most common measure taken was the drug treatment $(65,5 \%)$. Conclusion: The majority of treatment was classified as urgency. The non-traumatic causes were the most frequent diseases. São Geraldo Hospital plays an important role, as a public service, in treating ophthalmic urgencies of Belo Horizonte region and surrounding towns.

Keywords: Eye diseases/epidemiology; Emergencies; Conjunctivitis; Eye foreign bodies; Eye injuries

\footnotetext{
${ }^{1}$ Médico Oftalmologista, preceptor do Serviço de Urgência em Oftalmologia do Hospital São Geraldo/Hospital das Clínicas Universidade Federal de Minas Gerais - UFMG - Belo Horizonte (MG), Brasil;

${ }^{2}$ Médica Oftalmologista do Hospital São Geraldo, Hospital das Clínicas - Universidade Federal de Minas Gerais - UFMG - Belo Horizonte (MG), Brasil;

${ }^{3}$ Médica Oftalmologista graduada pela Faculdade de Medicina da Universidade Federal de Minas Gerais - UFMG - Belo Horizonte (MG), Brasil. Em exercício no Município de Diamantina (MG), Brasil;

${ }^{4}$ Médica Oftalmologista, realizando "fellowship" em glaucoma na Clínica de Olhos da Santa Casa de Belo Horizonte (MG), Brasil;

${ }^{5}$ Médico, Gestor do Instituto de Olhos do Hospital Universitário São José, Belo Horizonte (MG), Brasil.

Trabalho realizado no Hospital São Geraldo, Hospital das Clínicas da Universidade Federal de Minas Gerais - UFMG - Belo Horizonte (MG), Brasil
}

Os autores declaram inexistir conflitos de interesse

Recebido para publicação em: 20/10/2010 - Aceito para publicação em 30/6/2011 


\section{INTRODUÇÃO}

$\mathbf{0}$ Hospital São Geraldo (HSG), parte integrante do complexo Hospital das Clínicas da Universidade Federal de Minas Gerais, mantém uma parceria com a Secretaria Municipal de Saúde para o atendimento pelo Sistema Único de Saúde (SUS) de emergência e urgência em oftalmologia. O HSG que sempre prestou esse tipo de atendimento verificou que o número desses pacientes tem aumentado de forma considerável. Outros centros de referência em oftalmologia do país também têm observado este aumento ${ }^{(1-2)}$.

O presente estudo tem como objetivo analisar a natureza e a gravidade dos atendimentos em um serviço universitário de urgência em oftalmologia. Foi considerada a hipótese de que o atendimento eletivo em oftalmologia da rede pública na grande Belo Horizonte não atende à demanda. Consequentemente, a demanda reprimida procura o serviço de urgência do HSG na tentativa de uma consulta, tornando esse serviço prestador principalmente de atendimentos eletivos.

\section{Métodos}

Os dados foram obtidos através de um questionário diário de atendimento, aplicado diretamente ao paciente e preenchido pelo médico residente. O questionário contém informações sobre dia da semana, período diurno e noturno, idade, sexo, procedência e diagnóstico, segundo o Código Internacional de Doenças (CID), 10 revisão e condutas iniciais adotadas. Foram coletados e analisados dados do atendimento de todos os dias da semana, 24 horas por dia, no período de setembro de 2005 a janeiro de 2006 (total de cinco meses completos). Não houve triagem prévia em relação à demanda espontânea dos pacientes. Foram coletadas informações sobre 8.346 pacientes atendidos, dados que foram submetidos à análise estatística descritiva após serem armazenados no programa Excel.

Para fins de análise, os atendimentos foram classificados em eletivos, urgências e emergências. Consulta oftalmológica eletiva envolve o atendimento de afecções crônicas que não necessitam de tratamento imediato. As urgências oftalmológicas incluem afecções agudas que necessitam de tratamento imediato, porém não envolvem risco de perda visual permanente nem complicações que possam levar à morte. Já as emergências oftalmológicas incluem afecções agudas que necessitam de tratamento imediato e caso não tratadas podem evoluir para perda visual permanente e/ou amea-

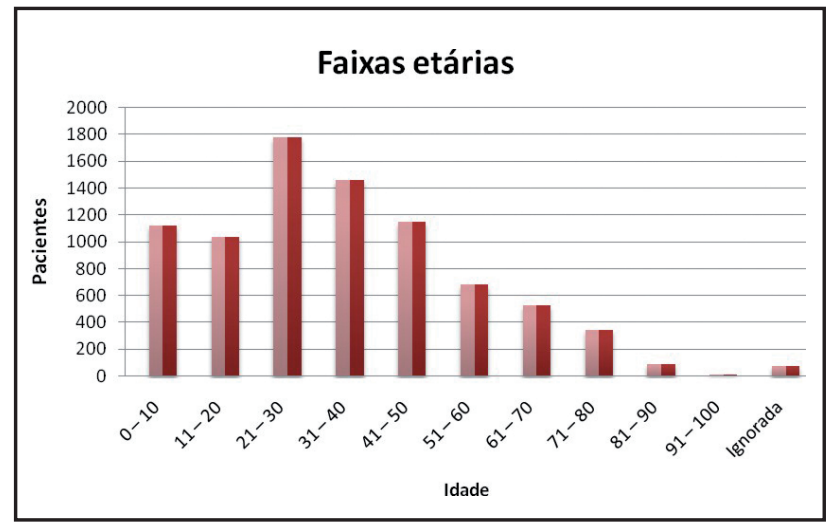

Figura 1: Faixas etárias

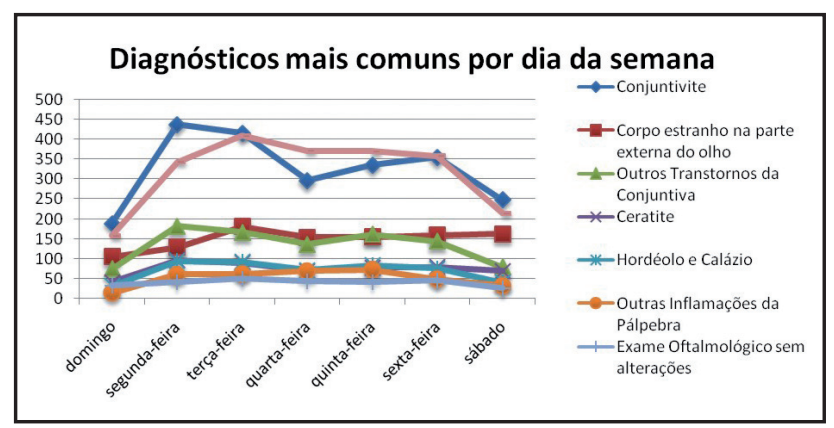

Figura 2: Diagnósticos mais comuns por dia da semana

çar a vida do paciente ${ }^{(3)}$.

O total de casos de conjuntivite atendidos às segundas e terças-feiras foi comparado ao total de casos nos demais dias da semana a partir do teste t-Student, uma vez que os dados seguem distribuição normal (verificada através do teste de Shapiro-Wilk). O mesmo procedimento foi utilizado para a comparação do total de casos de trauma ocular atendidos aos sábados e domingos com o total de casos atendidos de segunda a sexta-feira. Foi considerado estatisticamente significativo um valor $\mathrm{p}$ menor que 0.05 .

A ausência de preenchimento, por parte do médico, de algum campo do questionário da pesquisa excluiu da análise apenas este dado específico. Porém os demais dados do paciente, se preenchidos corretamente, foram analisados. $\mathrm{O}$ fornecimento de atestado médico foi registrado no trabalho como conduta médica, porém por julgarmos não se tratar de conduta terapêutica, optamos por excluí-lo da análise.

\section{Resultados}

Foram atendidos 8.346 pacientes, 3.819 mulheres $(45,79 \%)$ e 4.521 homens $(54,20 \%)$. A faixa etária va- 
Tabela 1

Diagnósticos mais comuns

\begin{tabular}{|c|c|c|}
\hline Diagnóstico & $\mathbf{n}$ & $\%$ \\
\hline Conjuntivite $^{1}$ & 2.275 & 27,91 \\
\hline Corpo estranho na parte externa do olho ${ }^{1}$ & 1.048 & 12,86 \\
\hline Outros transtornos da conjuntiva ${ }^{1}$ & 944 & 11,58 \\
\hline Ceratite $^{1}$ & 524 & 6,43 \\
\hline Hordéolo e Calázio ${ }^{1}$ & 491 & 6,02 \\
\hline Outras Inflamações da pálpebra ${ }^{1}$ & 363 & 4,45 \\
\hline Exame oftalmológico sem alterações & 283 & 3,47 \\
\hline Outros transtornos da córnea ${ }^{1}$ & 227 & 2,78 \\
\hline Traumatismo do olho e da órbita ocular² & 182 & 2,23 \\
\hline Queimadura e corrosão limitadas ao olho e seus anexos ${ }^{1}$ & 168 & 2,06 \\
\hline Iridociclite $^{1}$ & 134 & 1,64 \\
\hline Transtornos do aparelho lacrimal $^{1}$ & 128 & 1,57 \\
\hline Glaucoma agudo $^{2}$ & 128 & 1,57 \\
\hline Inflamação coriorretiniana ${ }^{2}$ & 111 & 1,36 \\
\hline Outros transtornos da pálpebra ${ }^{1}$ & 106 & 1,30 \\
\hline Demais diagnósticos & 1.039 & 12,75 \\
\hline Total & 8.151 & 100 \\
\hline
\end{tabular}

1 - Urgência oftalmológica; 2 - Emergência oftalmológica

Tabela 2

Distribuição dos casos de conjuntivite e traumatismo ocular ao longo dos dias da semana. Dados correspondentes ao total de casos durante o estudo nos respectivos dias

\begin{tabular}{|c|c|c|c|c|c|c|}
\hline \multicolumn{7}{|c|}{ Conjuntivite } \\
\hline Domingo & Segunda-feira & Terça-feira & Quarta-feira & Quinta-feira & Sexta-feira & Sábado \\
\hline 189 & 437 & 415 & 336 & 354 & 354 & 248 \\
\hline \multicolumn{7}{|c|}{ Traumatismo do olho e da órbita ocular } \\
\hline Domingo & Segunda-feira & Terça-feira & Quarta-feira & Quinta-feira & Sexta-feira & Sábado \\
\hline 20 & 24 & 33 & 29 & 30 & 26 & 20 \\
\hline
\end{tabular}

Tabela 3

\section{Condutas}

\begin{tabular}{lrr}
\hline Conduta & $\mathbf{n}$ & $\mathbf{\%}$ \\
\hline Tratamento medicamentoso (tópico e/ou sistêmico) & 8.453 & 65,5 \\
Orientação e observação & 1.557 & 12,1 \\
Retirada de corpo estranho de superfície ocular + curativo oclusivo & 1.131 & 8,8 \\
Encaminhamento às subespecialidades da Oftalmologia & 597 & 4,6 \\
Encaminhamento para consulta eletiva & 583 & 4,5 \\
Outras condutas & 161 & 1,2 \\
Propedêutica por imagem & 145 & 1,1 \\
Propedêutica laboratorial & 81 & 0,6 \\
Internação hospitalar para tratamento cirúrgico & 73 & 0,6 \\
Encaminhamento as outras especialidades médicas & 71 & 0,5 \\
Encaminhamento para cirurgia eletiva & 32 & $0,2 \%$ \\
Internação hospitalar para tratamento clínico oftalmológico & 15 & 0,1 \\
Cirurgia ambulatorial & 12 & 0,1 \\
Total & 12.911 & 100
\end{tabular}


riou de 0 a 100 anos com predominância entre 21 e 40 anos (39,5\%) (figura 1). Foram excluídos 06 pacientes da análise de sexo e 146 pacientes excluídos da análise de faixa etária, todos por informações incompletas.

A procedência foi na grande maioria de pacientes oriundos da grande Belo Horizonte (70,5\%). O grupo de ocupações mais frequente foi o de serviços gerais $(24,2 \%)$, termo utilizado para agrupar as ocupações de faxineiro, servente, porteiro, segurança e vigia. A segunda ocupação mais frequente foi as atividades domésticas $(13,7 \%)$ seguida por aposentados (10,5\%) e estudantes (7,6\%).

$\mathrm{O}$ diagnóstico mais frequente foi conjuntivite $(27,91 \%)$, incluindo conjuntivite infecciosa e alérgica, seguido por corpo estranho na parte externa do olho $(12,86 \%)$. Os traumatismos oculares associados a queimadura e corrosão do olho e anexos totalizaram 350 casos, representando 4,29\% dos diagnósticos (Tabela 1).

A figura 2 permite uma análise da incidência dos diagnósticos mais comuns ao longo dos dias da semana.

A tabela 2 apresenta a distribuição dos casos de conjuntivite e traumatismo ocular ao longo dos dias da semana. Há mais registros de casos de conjuntivite nas segundas e terças-feiras do que nos demais dias da semana (valor$\mathrm{p}=0,038)$ e menos registros de trauma ocular aos sábados e domingos comparado aos demais dias (valor- $p=0,024)$.

A conduta mais adotada no período do estudo foi o tratamento medicamentoso $(65.5 \%)$. O medicamento mais prescrito foi o colírio de antibiótico associado a anti-inflamatório esteróide, prescrito em $24,2 \%$ dos atendimentos (2.020 pacientes), seguido pelos lubrificantes oculares, $17,4 \%$ dos atendimentos. A segunda e terceira condutas mais adotadas foram a orientação/observação e retirada de corpo estranho da superfície ocular com realização de curativo (tabela 3 ). A tabela 3 contempla 12911 condutas para 8.346 pacientes devido a 4280 pacientes receberem mais de uma conduta, por exemplo, retirada de corpo estranho com realização de curativo e epitelizante de uso tópico. Casos em que foram prescritos dois tipos de medicamentos distintos também foram registrados como duas condutas distintas.

\section{Discussão}

A presente análise teve predominância de atendimentos de pacientes do sexo masculino, conforme resultados de outros estudos epidemiológicos ${ }^{(1,4-7)}$.

A faixa etária mais prevalente (21 a 40 anos) também se assemelha a resultados de trabalhos existentes ${ }^{(1,4,7-9)}$.

Quanto à classificação das morbidades houve uma incidência maior de causas não traumáticas, resultado diferente do apresentado em outros estudos, que demonstraram as afecções traumáticas como as mais frequentes em urgência oftalmológica ${ }^{(2,4-6,10,11)}$. Trabalhos estimam que aproximadamente $50 \%$ dos pacientes que procuram serviço de urgência oftalmológica apresentam história de trauma ${ }^{(10,11)}$. No presente estudo, os casos de traumatismo do globo e órbita ocular, incluindo queimaduras, representaram $4,29 \%$ dos atendimentos, percentual inferior ao encontrado em outros ${ }^{(2,4-6,10,11)}$. Esse dado pode ser explicado pela presença de um hospital referência em trauma (Hospital João XXIII) próximo ao Hospital São Geraldo. Os traumatismos oculares foram mais frequentes em dias úteis por provável relação com acidentes de trabalho, relação esta bem evidenciada em estudo epidemiológico realizado em Singapura ${ }^{(12)}$.

A morbidade mais freqüente foi a conjuntivite, achado que corrobora análise epidemiológica realizada em Sydney, na Austrália em $2001^{(7)}$ e publicado em 2005. Importante levar em consideração a coleta de dados do presente estudo que foi realizada em 2005.

Houve uma incidência menor de conjuntivites aos sábados e domingos e maior nas segundas e terças-feiras. Esse dado pode ser explicado pelo fato de determinados pacientes não procurarem atendimento médico aos sábados e domingos, seja por receio de encontrar o serviço médico fechado ou por dificuldade no transporte aos fins de semana. O paciente então procura o médico nas segundas ou terças-feiras, o que pode aumentar a incidência de determinadas afecções nesses dias específicos.

Nossa análise possui limitações que devem ser consideradas, dentre elas, a coleta de dados a partir de centro de referência em oftalmologia, podendo não receber pacientes com lesões mínimas tratadas em outras unidades de saúde e mesmo por não oftalmologista. Por outro lado, pacientes politraumatizados também não entraram nesses registros. Acidentes graves relacionados a automóveis ou a outras causas são normalmente referenciados ao Hospital João XXIII, por isso podem estar subestimados.

Houve uma predominância de casos classificados como urgência, foi solicitado marcação de consulta eletiva em 583 atendimentos, o que representa aproximadamente $7,0 \%$ dos pacientes registrados.

\section{ConClusão}

O presente estudo mostrou que a maioria dos atendimentos foi caracterizada como urgência oftalmológica. Esta análise anulou nossa hipótese de que o serviço de urgência do HSG seria prestador principalmente de aten- 
dimentos eletivos.

A coleta de dados foi realizada em 2005 e 2006, ou seja, retrata o perfil dos atendimentos ocorridos no Hospital São Geraldo há cinco anos. A conjuntivite foi a morbidade com maior incidência. Orientação adequada aos pacientes deve ser realizada para prevenção de novos casos e surtos infecciosos da doença. Corpo estranho na parte externa do olho foi a segunda morbidade mais frequente. Deve-se estimular o uso de equipamento de proteção capaz de evitar esse incidente.

Pode-se concluir que o HSG desempenha um papel importante no atendimento à urgência oftalmológica da rede pública.

São necessários novos estudos epidemiológicos a fim de compará-los aos nossos resultados.

\section{REFERÊNCIAS}

1. Campos Júnior JC. Perfil do atendimento oftalmológico de urgência. Rev Bras Oftalmol. 2004;63(2):89-91.

2. Leonor ACI, Dalfre JT, Moreira PB, Gaiotto Júnior OA. Emergências oftalmológicas em um hospital dia. Rev Bras Oftalmol. 2009;68(4):197-200.

3. Khare GD, Symons RC, Do DV. Common ophthalmic emergencies. Int J Clin Pract. 2008;62(11):1776-84.

4. Araújo AAS, Almeida DV, Araújo VM, Góes MR. Urgência oftalmológica: corpo estranho ocular ainda como principal causa. Arq Bras Oftalmol. 2002;65(2):223-7.
5. Pierre Filho PTP, Gomes PRP, Pierre ETL, Pinheiro Neto FB. Profile of ocular emergencies in a tertiary hospital from Northeast of Brazil. Rev Bras Oftalmol. 2010;69(1):12-7.

6. Cecchetti DFA, Cecchetti SAP, Nardy ACT, Carvalho SC, Rodrigues MLV, Rocha EM. Perfil clínico e epidemiológico das urgências oculares em pronto-socorro de referência. Arq Bras Oftalmol. 2008;71(5):635-8.

7. Kumar NL, Black D, McClellan K. Daytime presentations to a metropolitan ophthalmic emergency department. Clin Experiment Ophthalmol. 2005;33(6):586-92.

8. Sánchez Tocino H, Galindo Ferreiro A, Iglesias Cortiñas D, Galindo Alonso J, Fernández Muñoz M. Estudio epidemiológico de las urgencias oftalmológicas en un hospital general. Arch Soc Esp Oftalmol. 2004;79(9):425-32.

9. Diniz Filho A. Perfil do Serviço de Urgência Oftalmológica do Hospital São Geraldo no primeiro semestre de 2002 [monografia]. Belo Horizonte: Universidade Federal de Minas Gerais; 2004.

10. Chiapella AP, Rosenthal AR. One year in an eye casualty clinic. Br J Ophthalmol. 1985;69(11):865-70.

11. Vernon SA. Analysis of all new cases seen in a busy regional centre ophthalmic casualty department during 24-week period. J R Soc Med.1983;76(4):279-82.

12. Voon LW, See J, Wong TY. The epidemiology of ocular trauma in Singapore: perspective from the emergency service of a large tertiary hospital. Eye (Lond). 2001;15(Pt 1):75-81.

\author{
Endereço para correspondência \\ Frederico Braga Pereira \\ Rua Palmira, $n^{\circ} 343$ - apto 202 - Bairro Serra \\ CEP 30220-110 - Belo Horizonte (MG), Brasil \\ E-mail: fbragap@hotmail.com
}

